\title{
XA21-specific induction of stress-related genes following Xanthomonas infection of detached rice leaves
}

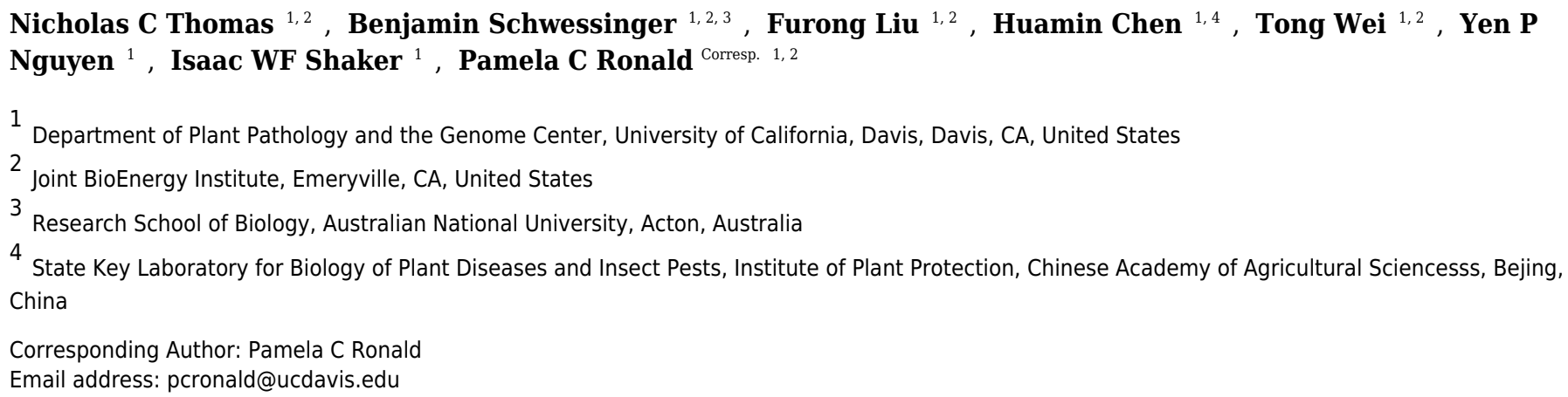

The rice $X A 21$ receptor kinase confers robust resistance to the bacterial pathogen Xanthomonas oryzae pv. oryzae (Xoo). We developed a detached leaf infection assay to quickly and reliably measure activation of the XA21-mediated immune response using genetic markers. We used RNA sequencing of elf18 treated EFR:XA21:GFP plants to identify candidate genes that could serve as markers for XA21 activation. From this analysis we identified 8 genes that are up-regulated in both in elf18 treated EFR:XA21:GFP rice leaves and $X 00$ infected XA21 rice leaves. These results provide a rapid and reliable method to assess bacterial-rice interactions. 
1 XA21-specific induction of stress-related genes following Xanthomonas infection of

2 detached rice leaves

3 Nicholas C Thomas ${ }^{1,2^{*}}$, Benjamin Schwessinger ${ }^{1,2,3^{*}}$, Furong Liu ${ }^{1,2}$, Huamin Chen ${ }^{1,4}$, Tong

4 Wei $^{1,2}$, Yen P Nguyen ${ }^{1}$, Isaac WF Shaker ${ }^{1}$, and Pamela C Ronald ${ }^{1,2}$

$5{ }^{1}$ Department of Plant Pathology and the Genome Center, University of California, Davis, Davis,

6 California, United States of America

$7 \quad{ }^{2}$ Joint BioEnergy Institute, Emeryville, CA 94608

$8{ }^{3}$ The Australian National University, Research School of Biology, Acton ACT 2601, Australia.

$9{ }^{4}$ State Key Laboratory for Biology of Plant Diseases and Insect Pests, Institute of Plant

10 Protection, Chinese Academy of Agricultural Sciences, Beijing 100193, China

$11^{*}$ These authors contributed equally to this work and are shown in reverse alphabetical order

12 Corresponding author:

13 Pamela C. Ronald ${ }^{1,2}$

14 One Shields Avenue

15 Davis, CA 95616, USA

16 E-mail address: pcronald@ucdavis.edu

18 Abstract

19 The rice XA21 receptor kinase confers robust resistance to the bacterial pathogen 20 Xanthomonas oryzae pv. oryzae (Xoo). We developed a detached leaf infection assay to quickly 21 and reliably measure activation of the XA21-mediated immune response using genetic markers.

22 We used RNA sequencing of elf18 treated EFR:XA21:GFP plants to identify candidate genes

23 that could serve as markers for XA21 activation. From this analysis we identified 8 genes that 
24 are up-regulated in both in elf18 treated EFR:XA21:GFP rice leaves and Xoo infected XA21 rice

25 leaves. These results provide a rapid and reliable method to assess bacterial-rice interactions.

26

\section{Introduction}

Plant immunity is mediated, in part, by cell surface immune receptors that recognize molecules produced by microbes. For example, the Arabidopsis FLS2 (lagellin Sensing 2) and EFR (Elongation Factor Tu Receptor) receptors recognize the flg22 peptide derived from bacterial flagellin and the elf18 peptide derived from elongation factor thermo-unstable (EF-Tu) protein, respectively (Gomez-Gomez and Boller 2000; Zipfel et al. 2006). The rice XA21 receptor recognizes the sulfated RaxX peptide (RaxX21-sY) derived from the RaxX protein produced by Xanthomonas oryzae pv. oryzae (Xoo) (Song et al. 1995; Pruitt et al. 2015; Wei et al. 2016). XA21, EFR, and FLS2 all contain extracellular leucine rich repeat (LRR), transmembrane, and intracellular non-RD (arginine-aspartic acid) kinase domains. These receptor domains are partially interchangeable. For example, the LRR domain from EFR can be fused to the transmembrane and intracellular domain of FLS2 to form a chimeric receptor that responds to elf18 treatments when transiently expressed in Nicotiana benthamiana and Arabidopsis thaliana (Albert et al. 2010). The EFR LRR can be fused to the transmembrane and intracellular domain of XA21 to form a chimeric receptor that responds to elf1 8 treatment and confers partial resistance to Xoo in transgenic rice lines (Schwessinger, Bahar, et al. 2015).

The availability of rapid and reliable assays that measure markers characteristic of immune response activation can help facilitate investigations of innate immune signaling. For example, immune signaling studies of FLS2 and EFR in Arabidopsis have been aided by the availability of rapid and reliable assays (Gomez-Gomez and Boller 2000; Zipfel et al. 2006; Chinchilla et al. 2007; Lu et al. 2010; Albert et al. 2010; Schulze et al. 2010; Schwessinger et al. 
48 2011; Sun et al. 2013; L. Li et al. 2014). In contrast, studies of the XA21-mediated immune

49 response have been limited by the lack of rapid assays and well-characterized genetic markers.

50 Typically, disease assessments are carried out by measuring lesions on rice leaves or by 51 assessing bacterial populations from infected leaves (Kauffman et al. 1973; Song et al. 1995; da 52 Silva et al. 2004; Park et al. 2008; Chen et al. 2014; Pruitt et al. 2015).

In this study we aimed to establish a rapid and efficient assay to monitor the XA21-

54 mediated immune response after bacterial infection. For this purpose, we employed the 55 EFR:XA21:GFP chimera composed of the EFR extracellular domain and the XA21 56 transmembrane and intracellular kinase domains, tagged with green fluorescent protein 57 (EFR:XA21:GFP) (Schwessinger, Bahar, et al. 2015). EFR:XA21:GFP transgenic rice plants are 58 partially resistant to Xoo and detached EFR:XA21:GFP leaves respond to elf18 with stress59 related gene induction, mitogen-activated protein kinase (MAPK) cascade activation, and 60 reactive oxygen species (ROS) production (Schwessinger, Bahar, et al. 2015). These results 61 indicate that plants expressing the EFR:XA21:GFP chimeric protein are appropriate for studies 62 to identify markers of resistance.

63 We used RNA sequencing (RNAseq) to identify genes differentially regulated in elf18 64 treated EFR:XA21:GFP rice. We then assessed if differentially regulated genes (DRGs) in elf18 65 treated EFR:XA21:GFP rice leaves were up-regulated in Xoo infected rice leaves expressing full66 length XA21, which are resistant to Xoo. We developed a rapid and reliable assay to analyze 67 gene expression in detached rice leaves inoculated with Xoo. We identified 8 DRGs from elf18 68 treated EFR:XA21:GFP rice that are also specifically up-regulated in detached XA21 rice leaves 69 infected with Xoo.

\section{Materials and Methods}


72 Plant growth, peptide and bacterial treatments of detached rice leaves

73 For peptide treatments, wild type (WT) Kitaake and progeny from line EFR:XA21:GFP-

74 3-8 (EFR residues 1-649 and XA21 residues 651-1025 called EFR:XA21:GFP in this study)

75 Kitaake rice leaves were harvested from plants grown in the greenhouse for 4.5 weeks

76 (Schwessinger, Bahar, et al. 2015). 1.5-2 cm leaf sections were collected from expanded adult

77 leaves using surgical grade scissors. Tissue from the leaf base and leaf tip was discarded.

78 Detached leaves were equilibrated overnight in 6-well Costar cell culture plates under

79 constitutive light (between $5-10 \mu \mathrm{mol} /\left(\mathrm{m}^{2 *} \mathrm{~s}\right)$ ) (Fig. S1). Peptide treatments were performed in 80 the morning and collected at the indicated times.

81 For bacterial inoculations, we used detached rice leaves harvested from 4-week old plants

82 grown using a hydroponic growth system as described previously (Pruitt et al. 2015) under a 83 light intensity of $280 \mu \mathrm{mol} /\left(\mathrm{m}^{2 *} \mathrm{~s}\right)$. Freshly harvested leaves from Kitaake and Ubi-Myc:XA21

84 Kitaake rice (called Myc:XA21 rice in this study) (Park et al. 2010) were cut into $1.5-2 \mathrm{~cm}$ 85 pieces and immediately (without overnight equilibration) floated on $10 \mathrm{mM} \mathrm{MgCl}_{2}$ solution for 86 mock treatments or $10 \mathrm{mM} \mathrm{MgCl}$ containing fresh Xoo cell suspensions at O.D.600 of 0.1 87 (approximately $1 \times 10^{7}$ cells $\mathrm{mL}^{-1}$ ). The samples were left overnight under constitutive light 88 (between 5-10 $\left.\mu \mathrm{mol} /\left(\mathrm{m}^{2 *} \mathrm{~s}\right)\right)$ and collected 24 hours post-inoculation (hpi). Leaves were floated 89 on approximately $1.5 \mathrm{~mL}$ Xoo cell suspension media in 6-well Corning Costar cell culture plates 90 (Fig. S1). The detached leaf infection assay allows a more uniform distribution, compared to the 91 scissor inoculation method (Kauffman et al. 1973), of Xoo inoculum by floating leaves on 92 bacterial suspensions. $R N A$ isolation and qPCR gene expression analysis for peptide treated and bacterial infected leaf 95 samples 
97 For tissue from greenhouse grown plants, RNA was extracted from powdered tissue using TRI

98 Reagent and precipitated with isopropanol. For tissue from hydroponically grown plants, RNA

99 was extracted using the Spectrum Plant Total RNA Kit from Sigma-Aldrich. RNA was DNase 100 treated using the TURBO DNase kit from Life Technologies. RNA concentrations were 101 normalized to the lowest sample concentration in each experiment. cDNA was synthesized from $1022 \mu \mathrm{g}$ of total RNA using the High Capacity cDNA Reverse Transcription Kit by Life 103 Technologies. Gene expression changes were determined by $\Delta \Delta \mathrm{Ct}$ method (Schmittgen and 104 Livak 2008) normalized to Actin (LOC_Os03g50885) and compared to mock treated samples.

105

Identification of genes differentially regulated in elf18 treated EFR:XA21:GFP rice using RNA

107

108

109

110

111

112

113

114

115

116

117

118

119

sequencing

Plant growth, leaf tissue isolation, and treatments were performed as described above. RNA was isolated from untreated Kitaake as well as untreated and elf18 treated EFR:XA21:GFP leaf tissue using the Spectrum Plant Total RNA Kit from Sigma-Aldrich and on-column DNase treated to remove genomic DNA contamination following the manufacturer's instructions. RNA was quantified using the Quant-IT Ribogreen RNA Assay Kit. Sequences were deposited to the NCBI Sequence Read Archive (BioProject ID PRJNA250865).

RNAseq libraries, sequencing, and reference alignment were performed as described previously (Schwessinger, Bahar, et al. 2015). Sample correlation between Kitaake and EFR:XA21:GFP replicates and differential gene expression analysis was performed using the Bioconductor 'edgeR' package for R (Robinson, McCarthy, and Smyth 2010; McCarthy, Chen, and Smyth 2012). 
120

121

122

123

124

125

126

127

128

129

130

131

132

133

134

135

136

137

138

139

140

141

142 identify genes differentially regulated during this response. We sequenced cDNA from

143 EFR:XA21:GFP leaves treated with $500 \mathrm{nM}$ elf18 for $0.5,1,3,6$, and $12 \mathrm{~h}$. We also included

Bacterial strains and generation of mutants

We generated a PXO99A $\triangle h r p A 1$ mutant in Philippine race 6 strain PXO99Az, a derivative of strain PXO99 (referred to as PXO99A in this study) (Salzberg et al. 2008). Xoo was grown in $10 \mathrm{~g}$ PSB (10 g Peptone (bacto-Peptone), $10 \mathrm{~g}$ Sucrose, $1 \mathrm{~g}$ sodium glutamate (glutamic acid, monosodium salt), final volume 1L, $\mathrm{pH}$ 7.0) or on PSA plates (PSB with 16g/L bacto-agar) at $28^{\circ} \mathrm{C}$. PXO99A $\triangle h r p A 1$ was generated by single crossover mutagenesis using the suicide vector pJP5603 (Penfold and Pemberton 1992). An approximately 500 base pair sequences within hrpAl was amplified using forward primer 5'CGGGGTACCGTGCTGCGTGATTTGTCCG-3' ${ }^{\prime}$ and reverse primer 5' CGCGGATCCTGACTTGGTCGATGCAGTCC-3' and cloned into the multiple cloning site of pJP5603 using the restriction enzyme sites KpnI and BamHI. PXO99A-competent cells were transformed with the suicide plasmids by electroporation and plated to PSA with kanamycin (50 $\mu \mathrm{g} / \mathrm{ml}$ ). PXO99A $\Delta h r p A 1$ colonies with kanamycin resistance were screened by PCR for colonies with single crossover events, which contain the vector disrupting the target gene. PXO99A $\operatorname{raxST}$ and PXO99A $\operatorname{rax} S T(\operatorname{rax} S T)$ complemented strains used in this study were described previously (Pruitt et al. 2015). PXO99A $\operatorname{raxST}$ evades XA21-mediated immunity while the complemented PXO99A $\operatorname{raxST}(\operatorname{rax} S T)$ strain does not.

\section{Results}

RNAseq analysis identifies 2212 genes that are differentially regulated in elf18 treated EFR:XA21:GFP rice leaves

We analyzed the transcriptomic profile of EFR:XA21:GFP rice lines treated with elf18 to 
144 untreated EFR:XA21:GFP and Kitaake as controls (Table S1). Multidimensional scaling of

145 pairwise biological coefficient of variance comparisons for each sample revealed that replicate

146 samples group together (Fig. 1A). This grouping of biological replicates demonstrates the overall

147 transcriptional similarity between each sample (Robinson, McCarthy, and Smyth 2010).

148 We identified 2212 genes that were differentially regulated in EFR:XA21:GFP rice

149 treated with elf1 8 compared with untreated $(0 \mathrm{~h})$ samples. Using a false discovery rate (FDR)

150 cutoff of 0.05 and absolute expression $\log$ fold change $(\operatorname{logFC})$ of 2 or greater, we previously

151 reported that the transcriptomic profile of untreated Kitaake compared to untreated

152 EFR:XA21:GFP did not differ significantly (Schwessinger, Bahar, et al. 2015). Over the

153 treatment time course, we identified 2212 DRGs (FDR $<0.05$, absolute $\log$ FC $>2$ ) using

154 untreated EFR:XA21:GFP at $0 \mathrm{~h}$ as a reference. The number of DRGs that overlap between the

155 elf18 treatment time points are summarized in Fig. 1B and File S1. Of the 2212 differentially

156 regulated genes, there were 1420 up-regulated and 792 down-regulated genes. The highest

157 number of DRGs (1504) was observed 6h post elf18 treatment. These results show that elf18

158 treated EFR:XA21:GFP rice express a substantially different set of genes over time compared to

159 untreated $(0 \mathrm{~h})$ samples.

160

161 Stress response related genes are up-regulated while photosynthesis related genes are 162 down-regulated in EFR:XA21:GFP rice treated with elf18

To examine the types of biological processes affected in elf18 treated EFR:XA21:GFP

164 rice, we analyzed GO term enrichment of DRGs using the AgriGo analysis tool (Du et al. 2010). 1204 out of 1420 of the up-regulated DRGs and 682 of the 806 down-regulated DRGs had GO annotations. An FDR of 0.05 or less was used to define significantly enriched terms compared to

167 the Michigan State University annotation reference as calculated by the AgriGo tool (Du et al. 
168 2010; Kawahara et al. 2013). Fig. 1C and File S2 summarize the most enriched GO terms in each

169 of the 3 major DRG clades. Clade 1 contains 1333 genes that are mostly up-regulated over time.

170 Genes from clade 1 are enriched for metabolic process (GO:0008152), response to stimulus

171 (GO:0050896) and response to stress (GO:0006950) GO terms (Fig. 1C). Clade 2 genes (122)

172 are up-regulated across all time points and are enriched for secondary metabolic process

173 (GO:0019748), metabolic process (GO:0008152) and response to stress (GO:0006950) GO terms

174 (Fig. 1C). Clade 3 consists of 757 genes that are mostly down-regulated in all timepoints.

175 Photosynthesis (GO:0015979) and response to abiotic stimulus (GO:0009628) are the most

176 enriched GO terms associated with clade 3 genes (Fig. 1C).

177

\section{qPCR validation of genes up-regulated in elf18 treated EFR:XA21:GFP plants}

We chose 23 DRGs from the elf18 treated EFR:XA21:GFP rice RNAseq dataset with relatively high $\log F C$ and low FDR values after 3, 6, and $12 \mathrm{~h}$ for detailed analysis. We first assessed if the 23 genes up-regulated in elf18 treated EFR:XA21:GFP could be validated by qPCR analysis. Eleven out of 23 DRGs were up-regulated in EFR:XA21:GFP rice leaves after elf18 treatment. Transcripts of the remaining 12 candidate genes were detectable by qPCR amplification but were not up-regulated in elf18 treated EFR:XA21:GFP leaves (File S3).

Establishment of bacterial infection assay of detached rice leaves

We established a detached leaf infection assay to test if genes identified in the Myc:XA21 rice. We observed bacterial ooze from the detached rice leaves three days after inoculation with Xoo strain PXO99A (Fig. 2). To further assess if Xoo infects rice leaves in our detached leaf infection assay, we measured the expression level of Os8N3 (LOC_Os08g42350), 
192 marker of successful infection (Yang, Sugio, and White 2006). For these experiments, we also 193 included a mutant PXO99A strain (PXO99A $\Delta h r p A 1)$ that is unable to infect rice as a control. 194 The hrpAl gene encodes a pilus protein that is essential for type III-secretion of effectors 195 required for host infection (Wengelnik et al. 1996). We observed that the PXO99A $\triangle$ hrpA1 Xoo 196 mutant is unable to infect Kitaake and Myc:XA21 rice plants (Fig. S2). Both WT Kitaake and 197 Myc:XA21 detached leaves express Os $8 N 3$ at higher levels compared to mock treatments $24 \mathrm{hpi}$ 198 with WT PXO99A, but not with PXO99A $\triangle$ hrpAl (Fig. 3). These results demonstrate that Xoo 199 infects detached rice leaves.

200 We next employed the detached leaf infection assay to examine the expression of the 201 stress-related marker $P R 10 b$ in Xoo infected Myc:XA21 rice leaves. Compared with mock 202 treated controls, $P R 10 b$ is up-regulated in flg22 treated rice, elf18 treated EFR:XA21:GFP rice 203 and Myc:XA21 rice treated with the RaxX21-sY (Chen et al. 2014; Schwessinger, Bahar, et al.

204 2015; Pruitt et al. 2015). Using qPCR, we detected significant up-regulation of PR10b expression 205 in Myc:XA21 rice leaves 24 hpi with PXO99A and PXO99A $4 h r p A 1 . P R 10 b$ up-regulation was 206 not observed in infected Kitaake leaves (Fig. 4). These results show that the detached leaf 207 infection assay can be used to assess XA21-mediated marker gene expression and also indicate 208 that RaxX expression or secretion is not affected by the $\Delta h r p A 1$ mutation.

Eight out of 11 genes induced in ef118 treated EFR:XA21:GFP rice are up-regulated in Xoo 211 infected XA21 rice

We next employed the detached leaf infection assay to identify genes up-regulated upon 213 Xoo infection of Myc:XA21 rice leaves. For these assays, we examined the gene expression of 214 the 11 genes validated by qPCR analysis of elf18 treated EFR:XA21:GFP rice described above. 215 We inoculated Kitake and Myc:XA21 rice with WT PXO99A, PXO99A $\operatorname{rax} S T$ mutants, and 
216 PXO99A 1 raxST complemented with $\operatorname{raxST}(\mathrm{PXO99A} \Delta \operatorname{rax} S T(\operatorname{rax} S T))$. Xoo strains carrying 217 mutations in $\operatorname{raxST}$ do not activate XA21-mediated immunity (da Silva et al. 2004; Pruitt et al. 218 2015). The expression of 8 of 11 genes was specifically up-regulated in detached Myc:XA21 rice 219 leaves 24 hpi with PXO99A and PXO99A $\operatorname{rax} S T(\operatorname{rax} S T)$ but not in Myc:XA21 rice leaves 220 infected with PXO99A $\operatorname{raxST}$ (Fig. 5 and File S3). The 8 validated marker genes encode a 221 putative subtilisin-like protein ( $\left.L O C_{-} O s 04 g 03100\right)$, a reticuline oxidase-like protein precursor 222 (LOC_Os06g35700), the decarboxylase OsTDC1 (LOC_Os08g04540) (Kang et al. 2007), a 223 peroxidase precursor (LOC_Os11g02100), RsOsPR10a (LOC_Os12g36830) (Hashimoto et al. 224 2004; Takeuchi et al. 2011), CYP71Z7 (LOC_Os02g36190) (W. Li et al. 2013), OsKO5 225 (LOC_Os06g37224) (Itoh et al. 2004), and one protein without a putative function 226 (LOC_Os $10 g 28299)$. The 3 remaining genes that were up-regulated in elf18 EFR:XA21:GFP 227 rice but not in Myc:XA21 rice leaves encode a isoflavone reductase (LOC_Os01g13610), a 228 subtilisin-like protein ( $\left.L O C_{-} O s 04 g 03100\right)$, and a reticuline oxidase-like protein precursor 229 (LOC_Os06g35700).

230

231 Discussion

232

In this study we identified 8 genes that are specifically up-regulated in both elf1 8 treated 233 EFR:XA21:GFP and Xoo infected detached Myc:XA21 rice leaves. At the time of these experiments, the activator of XA21, RaxX, had not yet been identified (Pruitt et al. 2015). We 235 therefore treated rice plants expressing the EFR:XA21:GFP chimera with elf18 to identify candidate marker genes because EFR:XA21:GFP rice are partially resistant to Xoo and respond 237 to elf18 treatments as described above in the introduction. Our results show that even though the EFR:XA21:GFP-mediated response does not confer robust resistance to Xoo (Schwessinger, Bahar, et al. 2015), similar genes are up-regulated during both EFR:XA21:GFP- and Myc:XA21- 
240 mediated responses (Fig. 5). Further studies are necessary to determine why the expression of

241 EFR:XA21:GFP in rice does not confer robust resistance to Xoo.

242 We show that stress-related gene induction of $P R 10 b$ in Myc:XA21 rice leaves is

243 maintained in plants inoculated with PXO99A $\Delta h r p A 1$ mutant strains. These results suggest that

244 RaxX expression, modification and secretion is not compromised by the $\Delta h r p A l$ mutation. These

245 results indicate that RaxX function is independent of type-III secretion mediated by $h r p A 1$. It

246 was previously reported that the $\operatorname{raxSTAB}$ operon, which encoded predicted components of a

247 type-I secretion system, was required for the processing and secretion of the XA21 elicitor (da

248 Silva et al. 2004). Our finding that RaxX function is independent of hrpAl-mediated type-III

249 secretion is consistent with the hypothesis that RaxX is a type I-secreted molecule (da Silva et al.

250 2004; Pruitt et al. 2015) and may provide insight into the largely unknown biological function of

251 RaxX.

252 The discovery of RaxX and the establishment of the detached leaf infection assay 253 described here provide useful tools for studying XA21-mediated immunity. XA21 activation can 254 be measured through ROS production and marker gene expression in detached leaves treated 255 with the RaxX21-sY peptide (Pruitt et al. 2015; Schwessinger, Li, et al. 2015). One advantage of 256 this approach is that researchers can study XA21-mediated immunity without working with Xoo. 257 Instead, researchers can activate XA21-mediated immunity by treating leaves with RaxX21-sY 258 peptide rather than Xoo. This strategy eliminates the need for select agent permits, which are 259 costly and time-consuming. The assay described in this study now allows researchers to use Xoo 260 infected plants to monitor XA21 activation by gene expression, which was previously only 261 possible using peptide treatments. This provides the benefit of monitoring bacterial induced 262 genes, such as $O s 8 N 3$ (Fig. 3). While we are not able to definitively assess resistance versus 
263 susceptibility to Xoo using this assay, we demonstrate that we can use gene expression to 264 monitor an immune response specifically mediated by XA21.

265 The detached leaf infection assay can also be used for other studies of bacterial-rice 266 interactions. For example, this system can be used to study rice immune responses conferred by 267 different resistance genes or induced by different bacterial strains. For example, the detached leaf 268 infection assay can be used to study the immune response conferred by other rice $\mathrm{Xa}$ genes 269 (Khan, Naeem, and Iqbal 2014) that confer resistance to Xoo such as $\mathrm{Xa3} / \mathrm{Xa26}$, which also 270 encodes a cell surface receptor kinase (Xiang et al. 2006; H.-J. Li et al. 2012). The detached leaf 271 infection assay can also be adapted to study immune responses to other races of Xoo (Niño-Liu,

272 Ronald, and Bogdanove 2006) or other Xanthomonas pathovars such as Xanthomonas oryzae pv. 273 oryzicola (Raymundo, Perez, and Co 1992; Niño-Liu, Ronald, and Bogdanove 2006).

274

\section{Acknowledgements}

276 We would like to thank Vasanth R. Singan, Rita Kuo, Mansi Chovatia, and Christopher Daum 277 from the Joint Genome Institute for their help with RNA sequencing. The United States

278 Government retains and the publisher, by accepting the article for publication, acknowledges that 279 the United States Government retains a non-exclusive, paid-up, irrevocable, world-wide license 280 to publish or reproduce the published form of this manuscript, or allow others to do so, for 281 United States Government purposes. 


\section{References}

283 Albert M., Jehle AK., Mueller K., Eisele C., Lipschis M., Felix G. 2010. Arabidopsis thaliana

284 pattern recognition receptors for bacterial elongation factor Tu and flagellin can be

285 combined to form functional chimeric receptors. The Journal of biological chemistry

$286 \quad 285: 19035-19042$.

287 Chen X., Zuo S., Schwessinger B., Chern M., Canlas PE., Ruan D., Zhou X., Wang J., Daudi A.,

288 Petzold CJ., Heazlewood JL., Ronald PC. 2014. An XA21-associated kinase (OsSERK2)

289 regulates immunity mediated by the XA21 and XA3 immune receptors. Molecular plant $290 \quad 7: 874-892$.

291 Chinchilla D., Zipfel C., Robatzek S., Kemmerling B., Nürnberger T., Jones JDG., Felix G., 292 Boller T. 2007. A flagellin-induced complex of the receptor FLS2 and BAK1 initiates plant 293 defence. Nature 448:497-500.

294 Du Z., Zhou X., Ling Y., Zhang Z., Su Z. 2010. agriGO: a GO analysis toolkit for the 295 agricultural community. Nucleic acids research 38:W64-70.

296 Gomez-Gomez L., Boller T. 2000. FLS2: An LRR Receptor-like kinase involved in the 297 perception of the bacterial elicitor flagellin in Arabidopsis. Molecular cell 5:1003-1011.

298 Hashimoto M., Kisseleva L., Sawa S., Furukawa T., Komatsu S., Koshiba T. 2004. A novel rice 299 PR10 protein, RSOsPR10, specifically induced in roots by biotic and abiotic stresses, 300 possibly via the jasmonic acid signaling pathway. Plant \& cell physiology 45:550-559.

301 Itoh H., Tatsumi T., Sakamoto T., Otomo K., Toyomasu T., Kitano H., Ashikari M., Ichihara S., 302 Matsuoka M. 2004. A rice semi-dwarf gene, Tan-Ginbozu (D35), encodes the gibberellin 303 biosynthesis enzyme, ent-kaurene oxidase. Plant molecular biology 54:533-547.

304 Kang S., Kang K., Lee K., Back K. 2007. Characterization of rice tryptophan decarboxylases and 305 their direct involvement in serotonin biosynthesis in transgenic rice. Planta 227:263-272. 
306 Kauffman HE., Reddy APK., Hsiek SPV., Marca SD. 1973. An improved technique for 307 evaluating resistance of race varieties to Xanthomonas oryzae. Plant Disease Managment $308 \quad$ Reports 57:537-541.

309 Kawahara Y., de la Bastide M., Hamilton JP., Kanamori H., McCombie WR., Ouyang S., 310 Schwartz DC., Tanaka T., Wu J., Zhou S., Childs KL., Davidson RM., Lin H., Quesada311 Ocampo L., Vaillancourt B., Sakai H., Lee SS., Kim J., Numa H., Itoh T., Buell CR., 312 Matsumoto T. 2013. Improvement of the Oryza sativa Nipponbare reference genome using next generation sequence and optical map data. Rice 6:4.

Khan MA., Naeem M., Iqbal M. 2014. Breeding approaches for bacterial leaf blight resistance in rice (Oryza sativa L.), current status and future directions. European journal of plant pathology / European Foundation for Plant Pathology 139:27-37.

Li H-J., Li X-H., Xiao J-H., Wing RA., Wang S-P. 2012. Ortholog alleles at Xa3/Xa26 locus confer conserved race-specific resistance against Xanthomonas oryzae in rice. Molecular plant-microbe interactions: MPMI 5:281-290.

Li W., Shao M., Yang J., Zhong W., Okada K., Yamane H., Qian G., Liu F. 2013. Oscyp71Z2 involves diterpenoid phytoalexin biosynthesis that contributes to bacterial blight resistance

Li L., Li M., Yu L., Zhou Z., Liang X., Liu Z., Cai G., Gao L., Zhang X., Wang Y., Chen S., in rice. Plant science: an international journal of experimental plant biology 207:98-107. Zhou J-M. 2014. The FLS2-associated kinase BIK1 directly phosphorylates the NADPH oxidase RbohD to control plant immunity. Cell host \& microbe 15:329-338.

Lu D., Wu S., Gao X., Zhang Y., Shan L., He P. 2010. A receptor-like cytoplasmic kinase, BIK1, associates with a flagellin receptor complex to initiate plant innate immunity. 

501.

\section{0}

McCarthy DJ., Chen Y., Smyth GK. 2012. Differential expression analysis of multifactor RNASeq experiments with respect to biological variation. Nucleic acids research 40:4288-4297.

Niño-Liu DO., Ronald PC., Bogdanove AJ. 2006. Xanthomonas oryzae pathovars: model pathogens of a model crop. Molecular plant pathology 7:303-324.

Park C-J., Peng Y., Chen X., Dardick C., Ruan D., Bart R., Canlas PE., Ronald PC. 2008. Rice $\mathrm{XB} 15$, a protein phosphatase $2 \mathrm{C}$, negatively regulates cell death and XA21-mediated innate immunity. PLoS biology 6:e231.

Park C-J., Lee S-W., Chern M., Sharma R., Canlas PE., Song M-Y., Jeon J-S., Ronald PC. 2010. Ectopic expression of rice Xa21 overcomes developmentally controlled resistance to Xanthomonas oryzae pv. oryzae. Plant science: an international journal of experimental plant biology 179:466-471.

Penfold RJ., Pemberton JM. 1992. An improved suicide vector for construction of chromosomal insertion mutations in bacteria. Gene 118:145-146.

Pruitt RN., Schwessinger B., Joe A., Thomas N., Liu F., Albert M., Robinson MR., Chan LJG., Luu DD., Chen H., Bahar O., Daudi A., De Vleesschauwer D., Caddell D., Zhang W., Zhao X., Li X., Heazlewood JL., Ruan D., Majumder D., Chern M., Kalbacher H., Midha S., Patil PB., Sonti RV., Petzold CJ., Liu CC., Brodbelt JS., Felix G., Ronald PC. 2015. The rice immune receptor XA21 recognizes a tyrosine-sulfated protein from a Gram-negative bacterium. Science Advances 1:e1500245.

Raymundo AK., Perez MT., Co S. 1992. Xanthomonas oryzae pv. oryzicola. In: 2. Asia-Pacific Biotechnology Congress, College, Laguna (Philippines), 22-24 May 1991. agris.fao.org,. 
351 Robinson MD., McCarthy DJ., Smyth GK. 2010. edgeR: a Bioconductor package for differential 352 expression analysis of digital gene expression data. Bioinformatics 26:139-140.

353 Salzberg SL., Sommer DD., Schatz MC., Phillippy AM., Rabinowicz PD., Tsuge S., Furutani A., 354 Ochiai H., Delcher AL., Kelley D., Madupu R., Puiu D., Radune D., Shumway M., Trapnell 355 C., Aparna G., Jha G., Pandey A., Patil PB., Ishihara H., Meyer DF., Szurek B., Verdier V., 356 Koebnik R., Dow JM., Ryan RP., Hirata H., Tsuyumu S., Won Lee S., Seo Y-S., Sriariyanum M., Ronald PC., Sonti RV., Van Sluys M-A., Leach JE., White FF., Bogdanove AJ. 2008. Genome sequence and rapid evolution of the rice pathogen Xanthomonas oryzae pv. oryzae PXO99A. BMC genomics 9:204.

Schmittgen TD., Livak KJ. 2008. Analyzing real-time PCR data by the comparative CT method. Nature protocols 3:1101-1108.

Schulze B., Mentzel T., Jehle AK., Mueller K., Beeler S., Boller T., Felix G., Chinchilla D. 2010. Rapid heteromerization and phosphorylation of ligand-activated plant transmembrane receptors and their associated kinase BAK1. The Journal of biological chemistry 285:94449451.

366

367

Schwessinger B., Roux M., Kadota Y., Ntoukakis V., Sklenar J., Jones A., Zipfel C. 2011. Phosphorylation-Dependent Differential Regulation of Plant Growth, Cell Death, and Innate Immunity by the Regulatory Receptor-Like Kinase BAK1. PLoS genetics 7:e1002046.

Schwessinger B., Bahar O., Thomas N., Holton N., Nekrasov V., Ruan D., Canlas PE., Daudi A., Petzold CJ., Singan VR., Kuo R., Chovatia M., Daum C., Heazlewood JL., Zipfel C., Ronald PC. 2015a. Transgenic Expression of the Dicotyledonous Pattern Recognition Receptor EFR in Rice Leads to Ligand-Dependent Activation of Defense Responses. PLoS pathogens 11:e1004809. 
374 Schwessinger B., Li X., Ellinghaus TL., Chan LJG., Wei T., Joe A., Thomas N., Pruitt R., 375 Adams PD., Chern MS., Petzold CJ., Liu CC., Ronald PC. 2015b. A second-generation 376 expression system for tyrosine-sulfated proteins and its application in crop protection. 377 Integrative Biology. DOI: 10.1039/C5IB00232J.

378 da Silva FG., Shen Y., Dardick C., Burdman S., Yadav RC., de Leon AL., Ronald PC. $2004 b$.

379 Bacterial genes involved in type I secretion and sulfation are required to elicit the rice 380 Xa21-mediated innate immune response. Molecular plant-microbe interactions: MPMI $381 \quad 17: 593-601$.

382 Song WY., Wang GL., Chen LL., Kim HS., Pi LY., Holsten T., Gardner J., Wang B., Zhai WX., 383 Zhu LH., Fauquet C., Ronald P. 1995. A receptor kinase-like protein encoded by the rice 384 disease resistance gene, Xa21. Science 270:1804-1806.

385 Sun Y., Li L., Macho AP., Han Z., Hu Z., Zipfel C., Zhou J-M., Chai J. 2013. Structural basis for 386 flg22-induced activation of the Arabidopsis FLS2-BAK1 immune complex. Science 387 $342: 624-628$.

Takeuchi K., Gyohda A., Tominaga M., Kawakatsu M., Hatakeyama A., Ishii N., Shimaya K., 389 Nishimura T., Riemann M., Nick P., Hashimoto M., Komano T., Endo A., Okamoto T., 390 Jikumaru Y., Kamiya Y., Terakawa T., Koshiba T. 2011. RSOsPR10 expression in response 391 to environmental stresses is regulated antagonistically by jasmonate/ethylene and salicylic 392 acid signaling pathways in rice roots. Plant \& cell physiology 52:1686-1696.

393 Wei T., Chern M., Liu F., Ronald PC. 2016. Suppression of bacterial infection in rice by 394 treatment with a sulfated peptide. Molecular plant pathology. DOI: 10.1111/mpp.12368. 
395 Wengelnik K., Marie C., Russel M., Bonas U. 1996. Expression and localization of HrpA1, a 396 protein of Xanthomonas campestris pv. vesicatoria essential for pathogenicity and induction 397 of the hypersensitive reaction. Journal of bacteriology 178:1061-1069.

398 Xiang Y., Cao Y., Xu C., Li X., Wang S. 2006. Xa3, conferring resistance for rice bacterial 399 blight and encoding a receptor kinase-like protein, is the same as Xa26. TAG. Theoretical $400 \quad$ and applied genetics. Theoretische und angewandte Genetik 113:1347-1355.

401 Yang B., Sugio A., White FF. 2006. Os8N3 is a host disease-susceptibility gene for bacterial 402 blight of rice. Proceedings of the National Academy of Sciences of the United States of $403 \quad$ America 103:10503-10508.

404 Zipfel C., Kunze G., Chinchilla D., Caniard A., Jones JDG., Boller T., Felix G. 2006. Perception 405 of the bacterial PAMP EF-Tu by the receptor EFR restricts Agrobacterium-mediated 406 transformation. Cell 125:749-760. 
A

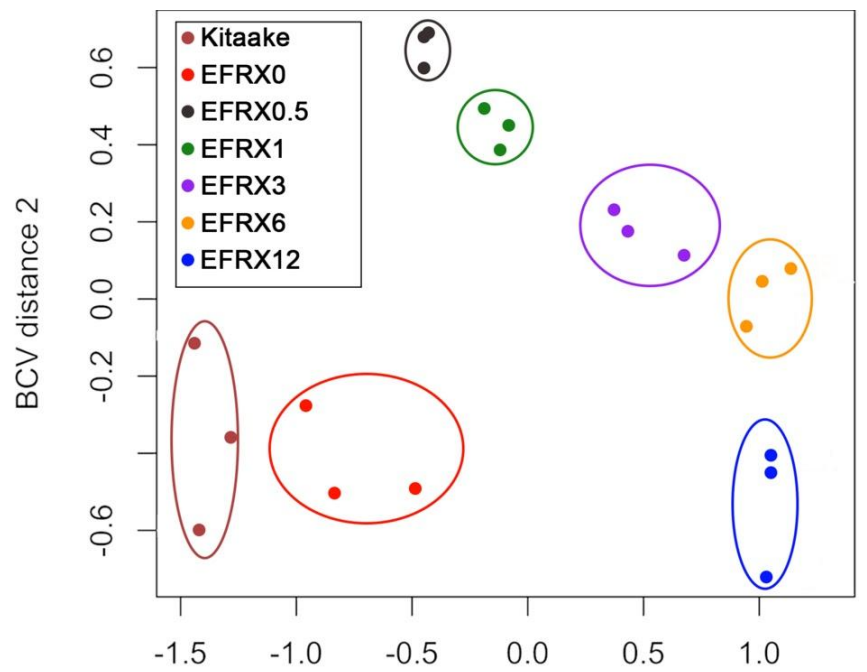

BCV distance 1
408

409

410

411

412

413

414

415
B

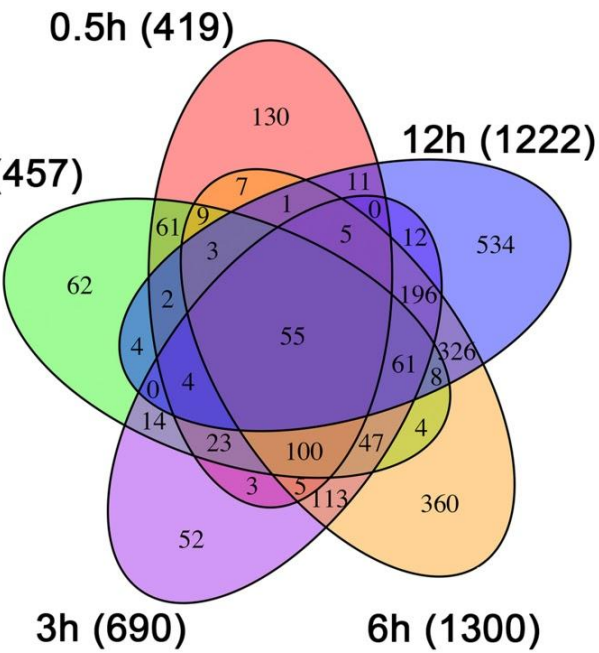

1

metabolic process response to stimulus response to stress protein modification process macromolecule modification

2

secondary metabolic process metabolic process response to stress response to stimulus

$\frac{3}{\text { photosynthesis }}$ response to abiotic stimulus
generation of precursor
metabolites
response to stimulus
Color Key
\begin{tabular}{|lll|}
\hline-10 & 0 & 10
\end{tabular}

Figure 1. The transcriptomic profile of elf18 treated EFR:XA21:GFP rice is enriched for stress response related and photosynthesis-related genes. (A) Multi-dimensional scaling comparing biological coefficients of variance between each sample. Samples labeled Kit0 are Kitaake rice leaf samples without treatment. Samples labeled Kitaake represent untreated Kitaake samples at $0 \mathrm{~h}$, EFRX represent EFR:XA21:GFP untreated samples (EFRX0) and samples treated with $500 \mathrm{nM}$ elf18 at 0.5h (EFRX0.5), $1 \mathrm{~h}$ (EFRX1), $3 \mathrm{~h}$ (EFRX3), $6 \mathrm{~h}$ (EFRX6), and $12 \mathrm{~h}$ (EFRX12). Groups of technical replicates are circled and sample color codes 
416 are indicated in upper left legend. (B) A five-way Venn diagram indicating number of total 417 (indicated in parentheses), unique and overlapping differentially regulated genes between time 418 points . (C) Heatmap representing expression levels of differentially regulated genes (DRGs) for 419 EFR:XA21:GFP samples treated with elf18 for indicated durations. The three major DRG clades, 420 determined by expression profile, are labeled 1,2 and 3 and are indicated to the right of the 421 heatmap. Significantly enriched gene ontology terms with a false discovery rate less than 0.5 , 422 compared to the reference, for each clade are shown on the right under the respective clade 423 number. The heatmap color key indicates $\log _{2}$ fold change values compared with untreated, 424 EFR:XA21:GFP 0h samples. 
Figure 2. Bacterial oozes from an infected rice leaf. Bacterial oozing (white arrowheads) was

427 observed from rice leaf xylem vessels three days post infection. This image shows detached 428 Kitaake rice leaves infected with PXO99A in a 6-well cell culture plate. Bacterial oozing was 429 consistently observed in Kitaake and Myc:XA21 detached leaves infected with PXO99A. Rice 430 leaves were collected from 4-week old, hydroponically grown plants and floated on Xoo cell 431 suspension media. 


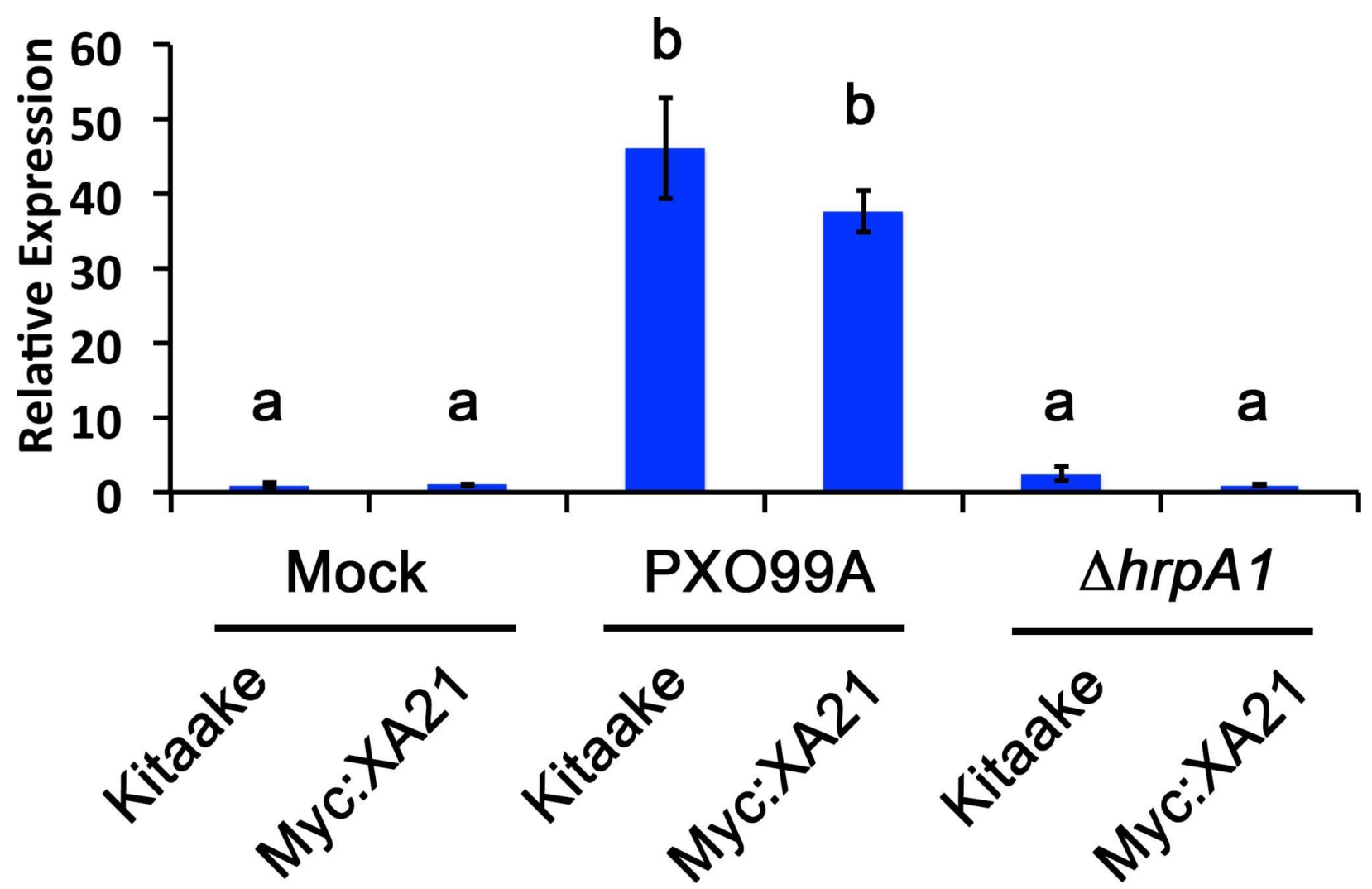

432

433

434

435

436

437 438

Figure 3. A marker gene of Xanthomonas infection, Os8N3, is up-regulated in PXO99A infected leaves. Os $8 N 3$ expression in detached Kitaake and Myc:XA21 rice leaves with $10 \mathrm{mM}$ $\mathrm{MgCl}_{2}$ mock treatment or infected with PXO99A or PXO99A $\Delta h r p A 1$ ( $\left.\Delta h r p A 1\right)$ at an O.D. 600 of 0.1 . Letters represent statistically significant differences between mean expression values $(\mathrm{p}<$

0.05 ) determined by using a Tukey-Kramer HSD test. This experiment was repeated three times with similar results. 


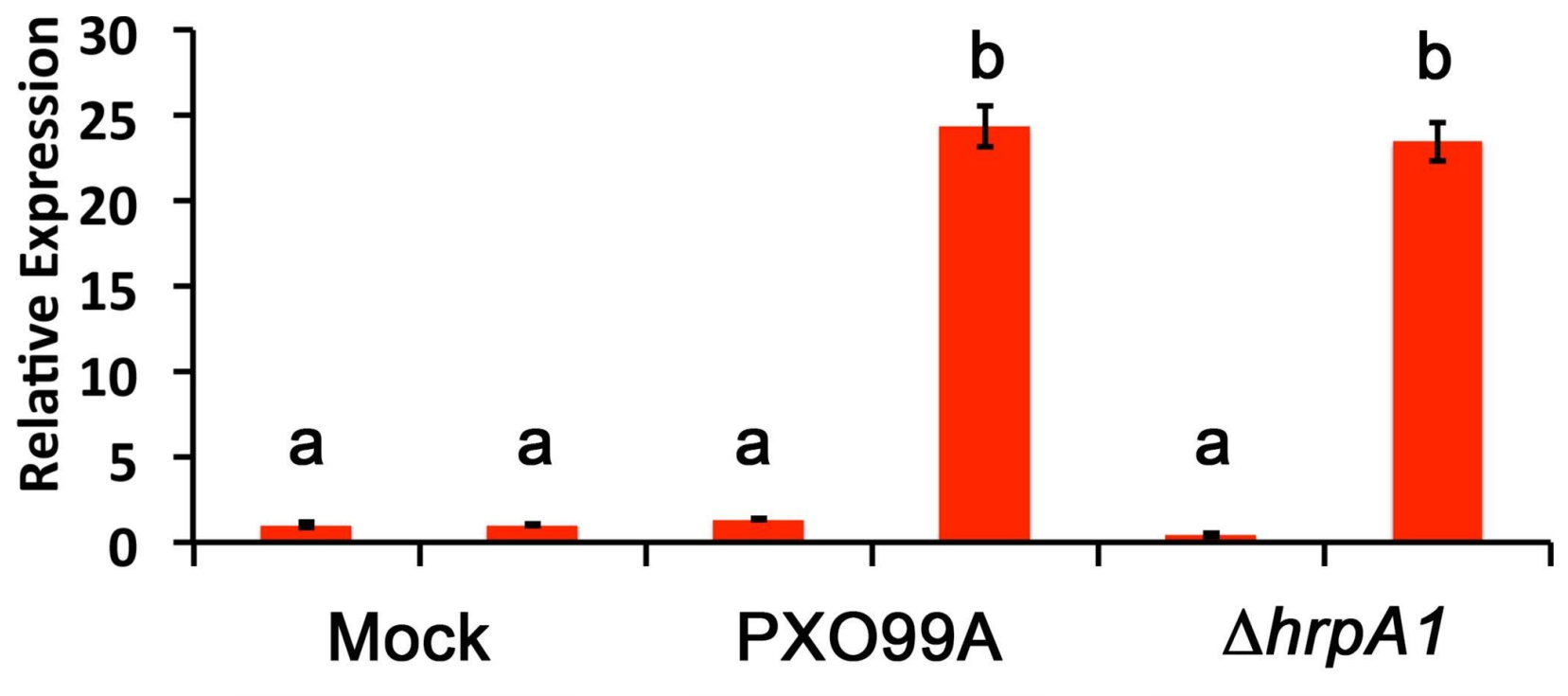

439

440

441

442

443

444

445
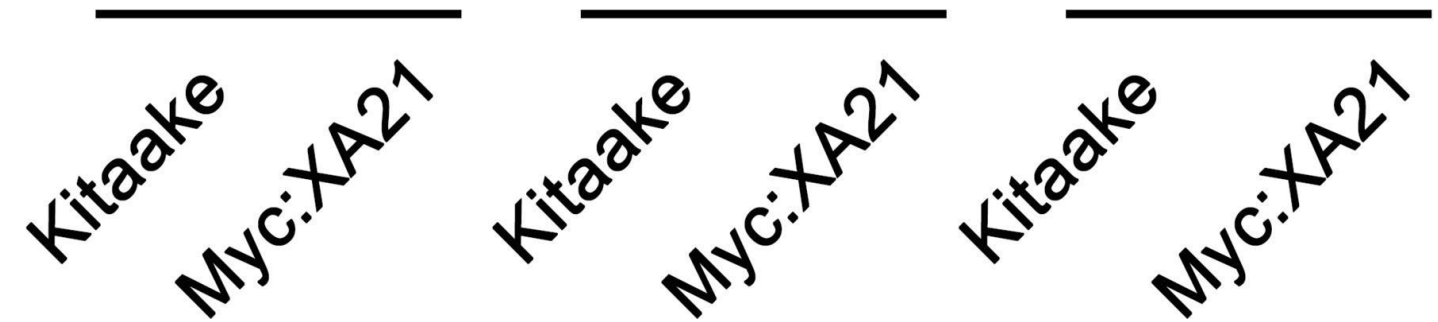

Figure 4. The stress-related marker gene $P R 10 b$ is up-regulated in Xanthomonas infected XA21 rice. $P R 10 b$ expression in detached Kitaake and Myc:XA21 rice leaves with $10 \mathrm{mM}$ $\mathrm{MgCl}_{2}$ mock treatment or infected with PXO99A or PXO99A $\Delta h r p A 1$ ( $\left.\Delta h r p A 1\right)$ at an O.D.600 of 0.1 . Letters represent statistically significant differences between mean expression values $(\mathrm{p}<$ 0.05 ) determined by using a Tukey-Kramer HSD test. This experiment was repeated three times with similar results. 

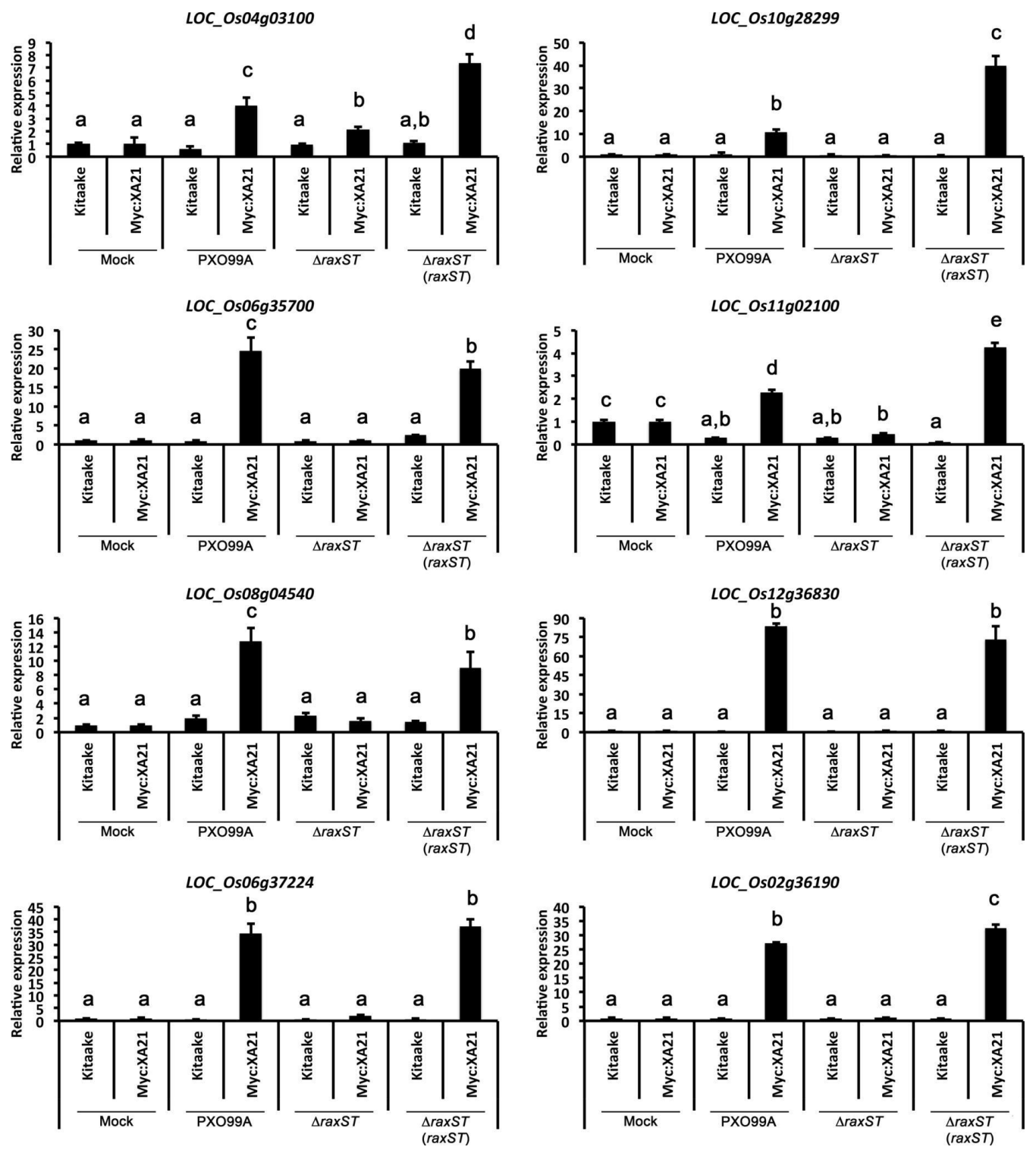

446

447

448

449

450

451

452

453

454

455

456

Figure 5. Eight marker genes are specifically up-regulated in detached rice leaves

undergoing the XA21-mediated immune response. Expression of 8 genes was measured in detached Kitaake and Myc:XA21 leaves infected with different Xoo strains. Mock samples were treated with $10 \mathrm{mM} \mathrm{MgCl}_{2}$. Xoo strains used for infection were WT PXO99A, a PXO99A $\Delta$ raxST mutant strain that evades XA21-mediated immunity ( $\triangle$ raxST), and the PXO99A $\Delta$ raxST mutant strain complemented with $\operatorname{raxST}(\operatorname{rax} S T(\operatorname{rax} S T)$ ). Expression levels are normalized to Actin then compared to mock treated samples. Bars indicate mean expression levels \pm standard deviation of three technical replicates. Letters represent statistically significant differences between mean expression values $(p<0.05)$ determined using a Tukey-Kramer HSD test. This experiment was repeated twice with similar results. 